\title{
Adaptive Eye-Camera Calibration for Head-Worn Devices
}

\author{
David Perra $^{1}$, Rohit Kumar Gupta ${ }^{2}$, Jan-Micheal Frahm² \\ ${ }^{1}$ Google Inc. \\ ${ }^{2}$ The University of North Carolina at Chapel Hill. \\ perra@google.com, \{rkgupta, jmf\}ecs.unc.edu
}

\begin{abstract}
We present a novel, continuous, locally optimal calibration scheme for use with head-worn devices. Current calibration schemes solve for a globally optimal model of the eye-device transformation by performing calibration on a per-user or once-per-use basis. However, these calibration schemes are impractical for real-world applications because they do not account for changes in calibration during the time of use. Our calibration scheme allows a headworn device to calculate a locally optimal eye-device transformation on demand by computing an optimal model from a local window of previous frames. By leveraging naturally occurring interest regions within the user's environment, our system can calibrate itself without the user's active participation. Experimental results demonstrate that our proposed calibration scheme outperforms the existing state of the art systems while being significantly less restrictive to the user and the environment.
\end{abstract}

\section{Introduction}

We are now at the verge of ubiquitously available consumer-grade head-wearable devices, with Google Glass serving as an early example. These devices enable new ways of interacting with the environment but also present challenges for meaningful interaction with the device. Currently, the most dominant mode of interaction with headworn devices is voice control, which allows for the triggering of preset tasks. However, this form of control is tedious for applications such as photography (for example, taking a controlled snapshot of a scene by zooming in on only a particular part of the scene; see Figure 1 for an example of a controlled photo). A natural alternative in controlling the camera's viewpoint is to allow the user's gaze to guide the photo-taking process. This becomes especially interesting now that there are cameras available that allow full digital zoom at the native sensor resolution by only selecting a part of the sensor for the photo; such a camera is already found on-board in the Nokia Lumia 1020. For these devices, it is
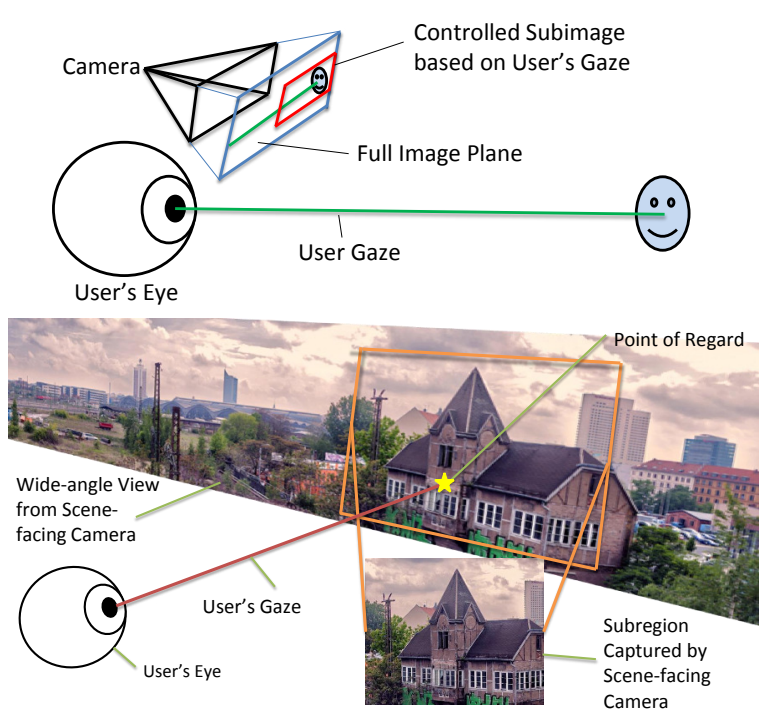

Figure 1: A diagram of a photography scenario in which the captured image's focal point is controlled by the user's gaze.

critical to select the correct sensor region based on the user's gaze at the time of capture. We propose a gaze tracking system for head-worn devices using a user-facing camera to extract the user's gaze. In addition, the proposed system can generally be used to determine the direction of user's attention by estimating their 3D point of regard (PoR) in the environment. The PoR can in turn be used for photography, safety notifications, analyzing user's social interaction, and other aids to the human visual system. Our PoR estimation technique is especially useful to a number of emerging research fields, including those focused upon human behavior and social interaction; these fields are forced to choose between overly cumbersome gaze tracking hardware (which may affect the subjects' behaviors or interactions) or attempting gaze estimation from face pose alone, losing out on gaze subtleties from eye-only gaze adjustments [18, 8].

Recent video-based eye tracking systems can be divided into two groups: 1. Appearance-based methods use the eye 


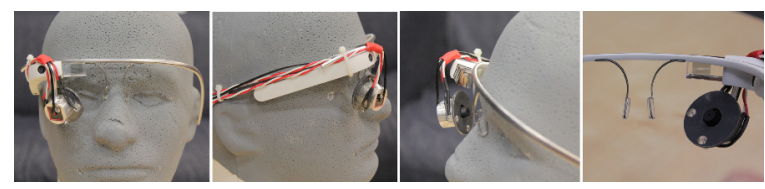

Figure 2: From left to right: 1) The front view of Google Glass paired with our custom eye tracking hardware. 2) The right-side view of the device. 3) A left-side view of the device. 4) A view of the device through the eyes of the user; the camera (center of the custom hardware) can focus as close as $\frac{1}{2}$ " away, minimizing the light output required from the LEDs to produce glints on the corneal surface.

image as a descriptor to obtain the gaze position [26, 13]. This method is sensitive to changes in illumination and eye/head position, making it prone to errors without careful calibration. 2. Shape-based methods track portions of the eye anatomy such as corneal reflection, pupil contour, and iris contour [15]. Corneal reflection and pupil contour methods require infrared ray (IR) active illumination [20,5].

User gaze information is undoubtedly a useful input mode for controlling head-worn devices, but it is detrimental to the user's experience when it is inaccurate. Calibration between the device and the user's eye is a critical but significant challenge. Take, for example, devices that assume a glasses-type form factor: even a small adjustment in the position of the device on the user's nose inherently changes its pose. Parameters of the eye which are used for gaze estimation also vary on a user-to-user basis. Thus, for gaze estimation to remain accurate at all times, it is necessary to constantly update the transformation between the device and the eye. To address this challenge, we propose a novel calibration scheme for the head-worn device to allow accurate gaze tracking with a simple head-worn system. The proposed calibration scheme leverages naturally occurring regions of interest instead of traditional calibration markers in order to generalize to arbitrary scenes. This calibration can be continuously performed during normal use of the head-worn device, as it does not require any active user participation. Our calibration operates over a window of recent frames in order to provide a highly accurate, locally optimal model for the current eye-device transformation.

\section{System Overview}

Our gaze detection system as shown in Figure 2 contains a user-facing camera that is used to capture user's eye movement. IR LEDs are positioned beside the camera to produce glints on the eye and also produce a dark-pupil effect that helps differentiate the pupil from the iris [15]. Our system also uses a scene-facing camera that captures the user's environment synchronously with the user-facing camera. These cameras are internally calibrated, and their views do

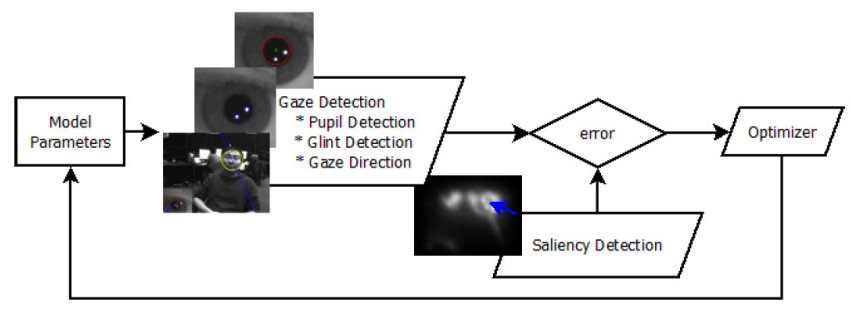

Figure 3: The proposed framework for eye-camera parameters estimation pipeline.

not overlap, similar to the system of Kumar et al. [9]. Our method (shown in Figure 3) then estimates the user's gaze from each pair of frames received from the above system. Adaptive thresholding is performed on the frames in order to filter out the IR reflections (glints) and the pupil from the rest of the eye. The Laplacian of the image is then used to calculate the pupil's convex hull. An ellipse is subsequently fitted to the hull in order to produce the final pupil boundary. This approach produces an accurate pupil estimate even if eyelashes, eyelids, or specular reflections slightly occlude the pupil. With two glints and the projected pupil center found in the eye image, along with a coarse approximation of the physical parameters of the system, we are able to successfully estimate the user's gaze.

Once the gaze is found in the coordinate system of the user-facing camera, it is projected upon the scene-facing camera's image plane. Ideally, the projected gaze would pass through the pixels of the scene image corresponding to the user's PoR. However, the projected gaze is likely to have errors for two reasons: 1) the user's eye parameters are initially unknown (initial estimates are used before the system is calibrated), and 2) the transformation between the eye and the device and the transformation between the two cameras are only initial approximations. To remedy this, we can perform an automatic calibration using naturally occurring regions of interest found within the scene image. Much research has focussed on obtaining good saliency maps that well approximate the points in an image at which an average user is inclined to look. Results obtained by Alnajar et al. [1] and Shi et al. [24] have been quite promising, and Chen et al. [2] have used these cues to obtain a probabilistic estimate of the user's gaze and its parameters by calibrating the cameras from the saliency maps obtained from the above methods and combining it with the 3D eye model.

\section{Related Work}

Traditionally, gaze estimation techniques predict a user's gaze in scenarios where they can move in front of a computer screen [15, 31, 14]. A significant portion of gaze estimation research is either focused upon improving humancomputer interaction or on advancing assistive technology 


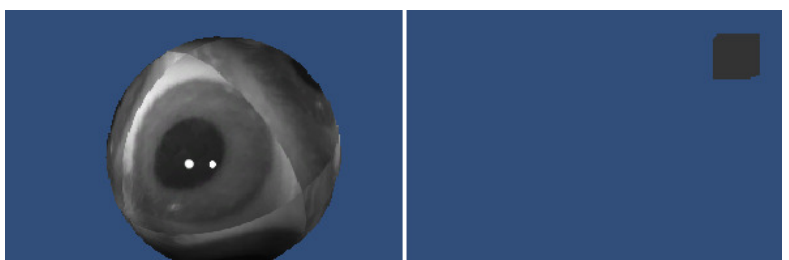

Figure 4: Simulated eye (left) and a Point of Regard square box object (right) in the scene which the simulated eye follows.

for the impaired [29, 10, 3, 11]. As wearable devices have become more widely used, gaze estimation has also been explored for systems with see-through displays [27, 17]; however, all these gaze tracking systems require a careful pre-calibration and rely upon a stable calibration throughout their use. For head-worn devices, the calibration typically changes during use as well as when the device is taken off or put on. It is not practical to have the user actively perform a calibration procedure every time the device's pose changes. In contrast to the existing state-of-the-art approaches, our technique performs a continuous calibration of the device in a simple way by utilizing the user's natural gaze in previous frames and observing salient areas of interest within the scene.

Hansen et al. [5] compared several different approaches, most of which estimate the user's point of regard (PoR). The PoR techniques presented in their paper map a user's gaze onto a computer screen that is in a fixed relative pose with respect to the user [15]. On the contrary, our approach finds the PoR by relating the user's gaze to automatically detected salient regions of interest within the scene, breaking the requirement for a known scene geometry and known user-to-camera settings.

Typically, the initial calibration required for accurate gaze tracking involves the user's active cooperation by looking at a number of predefined points [30, 16]. Sugano et al. [25] achieved an error-corrected two-eye gaze estimate by showing natural images/videos to the user and leveraging saliency maps to determine what object was being looked at by the user. Their results show that combining saliency metrics, including face detection, allows for better modeling of the human visual system. Our method takes this concept further by using salient areas found within the real world as an indication of the user's gaze direction during our calibration process.

Tsukada et al. [28] presented a system that determines the user's PoR by extracting the gaze from a single eye and leveraging an appearance code book for the gaze mapping. This appearance code book is very sensitive to the calibration, which is performed in a constrained environment and assumed to be constant throughout use. This assumption is not always valid due to configuration and environmental changes. In contrast, our method does not require a global calibration and is continuously recalibrating the configuration of the user with respect to the head-worn device .

Nakazawa et al. [16] demonstrated a gaze estimation system which projects a coded light pattern upon the scene using a multispectral LED array. Martinez et al. [13] inferred the gaze by relying upon appearance-based gaze estimation; they handled relative pose changes between the device and the user by estimating the eye-device transformation using a motion capture system in their testing environment. These techniques produce state-of-the-art results but rely upon specialized hardware that is not found in general environments.

Pirri et al. [21, 22] proposed a procedure for calibrating a scene-facing camera's pose with respect to the user's gaze. While effective, the technique's dependence upon artificial markers in the scene prevents generalization. Santner et al. [23] built upon the research done by Pirri et al. by combining 3D saliency with a dense reconstruction of the user's environment for the purposes of user localization. Aside from requiring the gaze from both eyes, their method assumes a static environment premapped by a Kinect sensor, and it thus is unable to handle dynamic scenes. In contrast, our proposed approach neither relies on static scenes nor requires any knowledge of scene depth.

In recent years, many researchers have devised models to generate better-quality saliency maps. Among these, Graph Based Visual Saliency (GBVS) [6], Adaptive Whitening Saliency (AWS) [12], and Image Signature (ImgSig) [7] usually exhibit the best performance. Recently, Shi et al. [24] proposed a Reverse Hierarchy Model (RHM) for predicting eye fixations. This method also provides a computational model for saliency detection in images.

Chen et al. [2] estimated the probability distributions of the eye parameters and eye gaze by combining an image saliency map with the 3D eye model. They used an incremental learning framework and avoided any personal calibration for the user. Our approach also uses a similar but different framework. Alnajar et al. [1] also developed an approach to auto-calibrate gaze estimators in an uncalibrated setup. Although this method used information from both eyes, additional stimulus signals were exploited to estimate gaze patterns obtained from specific users.

\section{Background}

Next, we introduce the basic concepts used in our approach for automatic continuous calibration of head-worn devices.

\subsection{Gullstrand Model of the Eye}

The Gullstrand model is a simplified representation of the human eye. This model is used in the geometric model- 


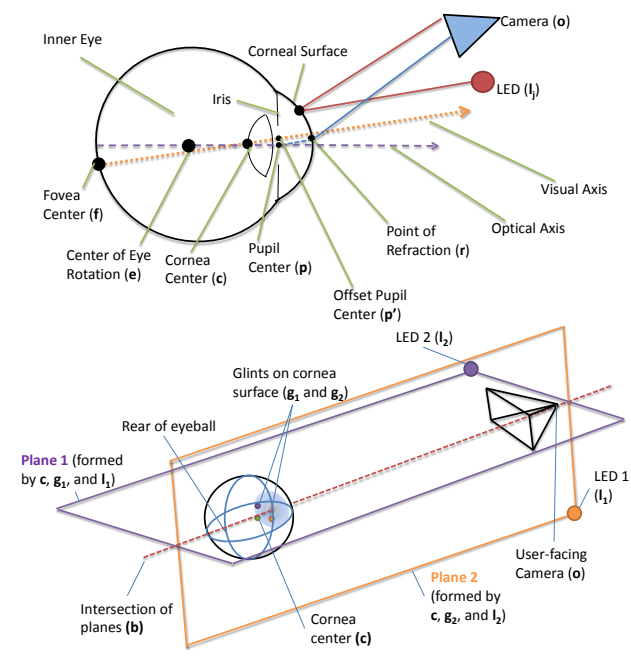

Figure 5: Top: A ray-traced diagram that, for illustrative purposes, depicts a geometric-based gaze estimation system for one light and one camera. The eye model pictured adheres to the Gullstrand model. Bottom: A 3D diagram depicting how the corneal center can be found from two planes, each defined by one LED, the corresponding glint, and the user-facing camera's position.

based gaze estimation technique that we employ in our approach.

A high-level illustration of the Gullstrand model is shown in Figure 5. For analytical purposes, the exterior corneal surface can be approximated by a spherical convex mirror. Additionally, the dominant refraction of light entering the eye is due to the air-corneal surface boundary [15]. The Gullstrand model defines the eye's optical axis as the vector which begins at the center of the cornea and passes through the center of the pupil. However, an individual's actual 3D PoR lies along their visual axis, since the fovea is offset on the rear of the eye [15]. The visual axis is related to the optical axis by two predefined rotations about the eye's corneal center. For our purposes, the visual axis is simply the user's normalized gaze vector.

The Gullstrand model of the eye also provides some initial estimates of the eye's structure. The model states that the cornea has an average radius of $R_{c}=7.7 \mathrm{~mm}$ and an average index of refraction of $\eta_{1}=1.3375$. The distance from the pupil to the corneal center is approximately $d_{c, p}=4.2$ $\mathrm{mm}$. Note that these parameters are user-specific and need to be optimized on an individual basis.

\subsection{Leveraging the Gullstrand Model of the Eye}

Our gaze estimation method is inspired by Guestrin et al. [4]. The goal of our technique is to estimate a user's visual axis in the coordinate system of a user-facing camera. The following discussion outlines the mathematical formulation of the gaze estimation problem. In the paper, we denote all $3 \mathrm{D}$ points in bold and lower case.

Consider a user-facing camera, o, positioned at the origin of the system. Additionally, consider a number of light sources, $\mathbf{l}_{\mathbf{j}}$, that are coplanar with the camera. Each light source, $\mathbf{l}_{\mathbf{j}}$, produces a reflection, $\mathbf{g}_{\mathbf{j}}$, on the user's corneal surface, known as a glint. Each glint intersects the image plane at a distinct point, $\mathbf{u}_{\mathbf{j}}$. The relationships between each of these points is presented in Figure 5. If the corneal center, $\mathbf{c}$, has radius $R_{c}$ then each glint, $\mathbf{g}_{\mathbf{j}}$, satisfies $\left\|\mathbf{g}_{\mathbf{j}}-\mathbf{c}\right\|=R_{c}$.

Guestrin derived, through the law of reflection, two additional constraints:

$$
\begin{array}{r}
\left(\mathbf{l}_{\mathbf{j}}-\mathbf{g}_{\mathbf{j}}\right) \cdot\left(\mathbf{g}_{\mathbf{j}}-\mathbf{c}\right) \cdot\left\|\mathbf{o}-\mathbf{g}_{\mathbf{j}}\right\|= \\
\left(\mathbf{o}-\mathbf{g}_{\mathbf{j}}\right) \cdot\left(\mathbf{g}_{\mathbf{j}}-\mathbf{c}\right) \cdot\left\|\mathbf{l}_{\mathbf{j}}-\mathbf{g}_{\mathbf{j}}\right\| \\
\left(\mathbf{l}_{\mathbf{j}}-\mathbf{o}\right) \times\left(\mathbf{g}_{\mathbf{j}}-\mathbf{o}\right) \cdot(\mathbf{c}-\mathbf{o})=0
\end{array}
$$

Equation (2) reveals that the corneal center is a point that lies on all planes defined by the camera, an LED, and that LED's glint. For a two-LED system, this implies that the corneal center lies on a line, $\mathbf{b}$, defined by the intersection of the two distinct planes. Refer to Figure 5 for an illustration of the intersecting planes. The corneal center, c, can be found by moving a distance of $d_{o, r}+R_{c}$ from the userfacing camera towards the user's eye along the line $\mathbf{b}$ (where $d_{o, r}$ is the current distance from the user-facing camera to the corneal surface). With the corneal center found, our task becomes finding the pupil center, p. Snell's law provides a constraint that allows us to solve for the pupil center from the positions of the user-facing camera and the glints, $\mathbf{u}_{\mathbf{j}}$, provided that we first find the point of refraction on the aircornea boundary, $\mathbf{r}$ :

$$
\begin{array}{r}
\eta_{1} \cdot\|(\mathbf{r}-\mathbf{c}) \times(\mathbf{p}-\mathbf{r})\| \cdot\|\mathbf{o}-\mathbf{r}\|= \\
\|(\mathbf{r}-\mathbf{c}) \times(\mathbf{o}-\mathbf{r})\| \cdot\|\mathbf{p}-\mathbf{r}\|
\end{array}
$$

Solving Equation (3) for the pupil center allows us to find the user's optical axis and, subsequently, to compute the visual axis. We approach this solution by temporarily changing the coordinate system to that shown in Figure 6 and solving for the pupil center with a sampling-based approach. This is opposed to the convention made by Guestrin et al. [4], who placed the eye's center of rotation, $\mathbf{e}$, as the center of the new coordinate system. Placing the corneal center at the origin of the system reduces the total number of coordinate system changes and provides a more convenient way to calculate the visual axis. The optical axis is associated with angles $\theta$ and $\phi$. The Gullstrand model of the eye dictates that the visual axis is approximately \pm 5 degrees to the side of the optical axis of the eye (call this angle $\alpha$ ), and about \pm 1.5 degrees above it (call this angle $\beta$ ). The equation for calculating the values of $\theta$ and $\phi$ is derived 


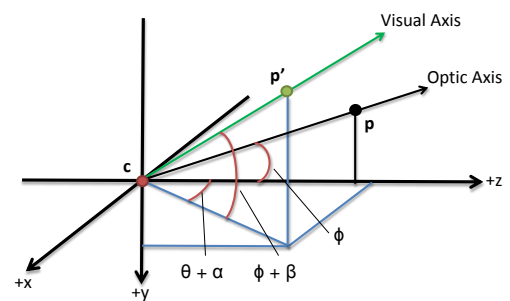

Figure 6: A depiction of the coordinate system used for the calculation of the visual axis.

from Figure 6 and is given by Equation (4)

$$
\frac{\mathbf{p}-\mathbf{c}}{\|\mathbf{p}-\mathbf{c}\|}=\left[\begin{array}{c}
\cos (\phi) \sin (\theta) \\
\sin (\phi) \\
-\cos (\phi) \cos (\theta)
\end{array}\right]
$$

The visual axis can be found by offsetting the pupil center, $\mathbf{p}$, to a nearby, adjusted pupil center, $\mathbf{p}^{\prime}$. Figure 6 depicts this offset. The adjusted pupil center is chosen to be a distance of $d_{c, p}$ away from the corneal center and must lie on the line passing through the corneal center, $\mathbf{c}$, and the center of the fovea, $\mathbf{f}$. Guestrin presented a method for calculating the offset pupil center $\mathbf{p}^{\prime}$ using the user-specific angles $\alpha$ and $\beta$. Finding the ray originating at the corneal center and passing through the adjusted pupil center yields the visual axis. This is the ray that is projected upon the scenecamera's image plane when determining the user's 3D PoR.

\section{Locally Optimal Eye-Camera Calibration}

To accommodate the changes in the eye-device transformation during its usage, we augment the user-specific parameters of the Gullstrand model by the device specific camera calibration parameters representing its degree of freedom. We expect that users will subconsciously fixate upon salient regions of the environment for multiple frames, meaning that the eye-device transformation will remain approximately constant for several frames. To leverage this observation, we optimize over a small window of recent frames and subsequently apply these locally optimal parameters to estimate gaze direction for next frames. We accomplish this by projecting the user's visual axis onto the image of the scene using an estimate of the current eye-device transformation. This effectively establishes an epipolar geometry between the eye and the camera. Then, by correcting each frame's gaze to the image's nearest calibration area of interest, we are able to find an eye-camera transformation that is optimal for all frames within the window of frames. This eye-camera transformation is then used to estimate gaze errors in the frames that occur immediately after the calibration window. When performed continuously or at high enough frequency, these calibration updates reduce the gaze projection error even as the eye-device relationship changes during use. Moreover, the continuous adjustment of the calibration accounts for the approximation error of the model due to the continuous re-estimation of the eyecamera parameters. The latter cannot be achieved with standard calibration techniques given that they estimate a global model whereas our proposed method effectively estimates a local model using the global model in short range of frames.

\subsection{Problem Description}

The user's estimated gaze must be accurately related to their PoR within the scene. Because head-worn devices tend to move about a user's face during typical use, a local calibration scheme is desirable. To accomplish this, the system must be capable of accurately estimating an optimal parametric model of the eye-device relationship at any time, $t$. We define model optimality to mean that, when projected upon the scene-facing camera's image plane, the Euclidian distance between the visual axis and the nearest calibration area of interest in the image plane is minimized at time $t$.

Recall that the Gullstrand model of the eye provides six main parameters to optimize over: the corneal radius, $R_{c}$, the eye's index of refraction, $\eta_{1}$, the distance from the pupil center to the corneal center of the cornea, $d_{c, p}$, the distance between the user-facing camera and the eye, $d_{o, r}$, and the two angular offsets used to find the visual axis from the optic axis: $\alpha$ and $\beta$. Refer to Figure 5 and Figure 6 for the relationships between each of these parameters. In addition, given the internal camera calibration the head-worn device has six degrees of freedom itself: three dimensions of translation $(x, y$, and $z)$ and three dimensions of rotation $(\phi, \theta$, and $\psi$ ). Hence, the parameters of an eye-camera model at a time $t$ can be described as

$$
P_{t}=<x, y, z, \phi, \theta, \psi, d_{o, r}, R_{c}, d_{c, p}, \eta_{1}, \alpha, \beta>
$$

\subsection{Salient Regions of Interest}

In order to make our system less cumbersome and more practical, we cannot rely upon markers placed throughout the user's environment for calibration. Hence, our system leverages salient features in the environment and it can automatically calibrate itself using gaze data collected during normal operation of the device. Combined with our continuous calibration approach, this yields all the benefits of a calibrated system while never interrupting or inconveniencing the user with manual device calibration.

Recently, much work has been done to improve the detection of salient regions [12, 7, 24, 6] in an image that closely correspond to the points where an average user is most likely inclined to look. Our system does not have any knowledge of the 3D geometry of the user's environment so it relies on a 2D saliency map of the scene in order to pick out these interest areas for calibration. 

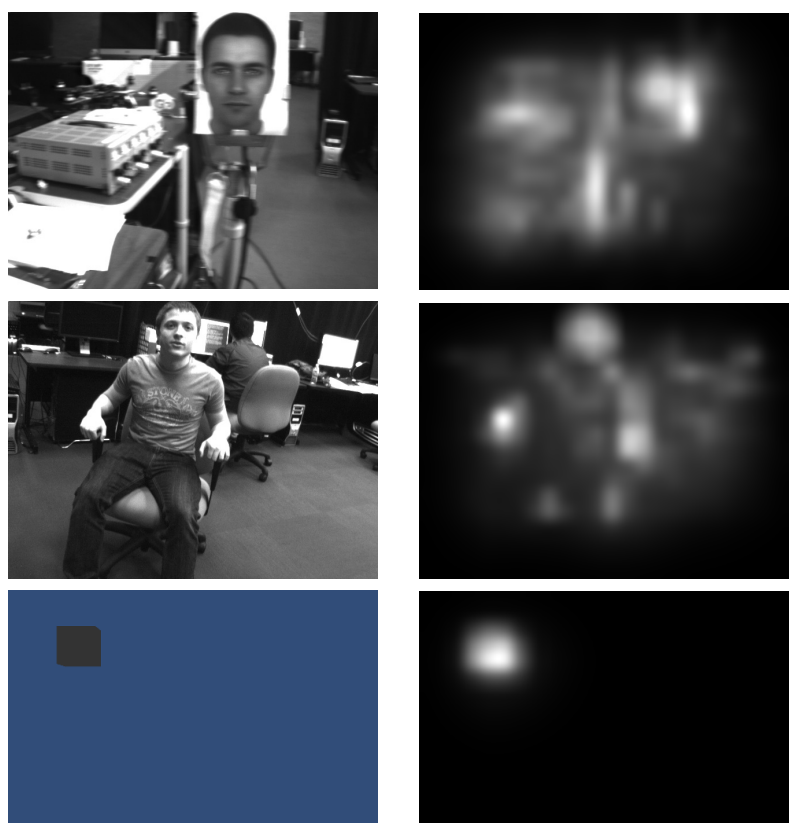

Figure 7: Input scene images (2 real environment, 1 simulation) and corresponding Saliency maps obtained by combination of GBVS and face detection.

Given a video stream of the user's eye, $U$, and scene facing camera, $S$, the saliency map, $M_{j}$, can be found for frame $S_{j}$ [25]. The user's projected visual axis, $v_{j}$, is found by obtaining the visual axis of the eye from $j^{\text {th }}$ frame of $U$ using the method described in Section 4.2 and projecting into frame $S_{j}$. We then threshold $M_{j}$ to find the set of most salient blobs of pixels, $S=\left\{s_{1}, \cdots, s_{n}\right\}$ for that frame. In our system, interest point $s_{j} \in S$ for an estimated gaze direction of $v_{j}$ is obtained simply by taking its nearest interest point from set $S$ obtained on $M_{j}$.

\subsection{Approach}

Next, we introduce the calibration equation for the eyecamera transformation. The eye-camera relationship at time $t$, described by the parametric model $P_{t}$ (Eq.5), allows for direct calibration between the user's eye and the scenefacing camera without having to know the exact extrinsic relationship between the user and scene-facing cameras. Our technique only assumes that the cameras are intrinsically calibrated; the only a priori knowledge that is required about the cameras' extrinsic relationship is a rough estimate of their transformation. Ordinarily, this extrinsic relationship could be found through mirror-based calibration techniques for cameras with non-overlapping views (for example, the algorithm proposed by Kumar et al. [9]). However, since our calibration scheme directly relates the visual axis with points on the scene-camera's image plane, accurate extrinsic camera calibration is unnecessary.
However, a user's fixation upon a region of the scene causes the user's visual axis to have frame-to-frame spatiotemporal constraints. By considering the set of possible models over the window, we can disambiguate the locally optimal solution. This locally optimal parametric solution, $P_{t}$, is then accurate for the current eye-device model at time $t$ and for small perturbations around that model (i.e. temporally close frames). $P_{t}$ is assumed to be constant over the window from which it is derived.

In order to optimize the eye-camera transformation, and to ensure that our calibration remains causal, our system minimizes the sum of absolute differences in the $x$ and $y$ directions between the projected visual axis, $v_{j}$, and the nearby salient interest point, $s_{j}$, for all frames within a short window of frames preceding time $t$. For a window of size $k$, the cost function, $C\left(p_{t}\right)$ can be written as:

$$
C\left(p_{t}\right)=\left[\begin{array}{c}
d_{x}\left(v_{t-1}, s_{t-1}\right) \\
d_{y}\left(v_{t-1}, s_{t-1}\right) \\
d_{x}\left(v_{t-2}, s_{t-2}\right) \\
d_{y}\left(v_{t-2}, s_{t-2}\right) \\
\ldots \\
d_{x}\left(v_{t-k}, s_{t-k}\right) \\
d_{y}\left(v_{t-k}, s_{t-k}\right)
\end{array}\right]
$$

where $d_{x}\left(v_{j}, s_{j}\right)$ is the absolute difference in the $x$-direction and $d_{y}\left(v_{j}, s_{j}\right)$ is the absolute difference in the $y$-direction for the projection of point $s_{j}$ on the gaze direction $v_{j}$.

We obtain the locally optimal solution, $P_{t}$, by solving the following least-squares optimization problem:

$$
P_{t}=\underset{p_{t}}{\operatorname{argmin}} \sum_{j=1}^{k} C_{j}\left(p_{t}\right)^{2}
$$

Optimizing over the eye-camera model parameters in the window yields an estimate of the locally optimal parametric model, $P_{t}$, at time $t$. The resulting parametric model effectively maximizes calibration accuracy while minimizing gaze estimation error. $P_{t}$ is further used as an initial estimate of the model parameters for the next window.

An important part of this approach is determining a good value of the window size $k$ for this optimization. As $k$ increases, the optimization approaches a single globally optimal eye-camera model. In our experiments, we study the performance of our system over a range of window sizes.

\section{Experimental Results}

Next, we evaluate the accuracy of our proposed local eye-camera calibration system. In all of the following experiments, the head-worn device's calibration was initialized with the initial eye parameters and pose parameters from the Gullstrand model (see Section 4.1) which roughly aligns the user-facing camera with the user's right eye. We 
use this generic setup to show that our system can adapt to any feasible head-device relationship.

Datasets. The performance of the proposed adaptive eye-camera calibration framework was evaluated on 10 datasets (5 human subjects and 5 simulations). Each dataset consists of 150-200 frames obtained from the user- and scene-facing camera. The scene-facing camera observed a point of regard approximately $1.1-1.5$ meters away from the participant. To limit the influence of confounds in the gaze error calculation, the users were instructed to look at the PoR and move their head in a circular fashion. For the human subject trials, the PoR was the face of another individual, seated at the same head level as the user. We used the specific class of faces for the salient region detection, but this setup could easily be generalized using saliency maps $[12,7,24]$, which would also impose similar constraints for the eye-camera transformation. All experimental setups have a known distance between the user and the calibration point, which is unknown to the system and is used only for determining our estimated gaze error. For all experiments the head-worn device was loosely attached to user's head to allow natural movement during use. For quantitative evaluation, we created a simulation of the whole system (see Figure 4), which generates image frames for an eye that follows an object moving along a specified path in the 3D world. The simulated data also contains a small, smooth perturbations of the camera pose, modeling real-world changes in the eye-camera relationship. This approach helps remove the bias when the user is looking at a specified PoR, also it provides a good error measurement. This model serves as a ground truth to check the accuracy of the system, as we have the labeled data for the gaze direction. This allows us to obtain accurate gaze estimation errors for our system as the ground truth is known.

Parameters. Our general eye-camera model has 12 unknown parameters at any time $t$ (see Section 5.1). However, in our experiments, we fixed the refractive index $\eta_{1}$ to a value of 1.3375 and the cornea-to-pupil distance $d_{c, p}$ to 4.5 $\mathrm{mm}$. As a result, we have 10 unknowns in our system that are estimated from a window of $k$ previous frames using the approach specified in previous section.

After finding the locally optimal model for time $t$ over the window of frames at $t-1$ to $t-k$, we analyze their accuracies; each window serves as an independent calibration set. Results for each experiment are presented as a graph (see Figure 8 and Figure 9) with three criteria plotted: 1) in blue, the re-projection error from applying the locally optimal model to the window that produced it (calibration error), 2) in red, the error produced at time $t$ when applying the locally optimal model (next-frame error), and 3 ) in green, the mean error produced when the locally optimal model is applied to the next five frames. We have also studied the behavior of window size $k$ over the accuracy of the system (Tables 1 and 2).

Table 1: Observed mean and standard deviation error (degrees) over all windows for a fixed window size $k$ (simulated dataset)

\begin{tabular}{|l|c|c|c|c|c|c|}
\hline$k$ & 5 & 10 & 15 & 20 & 25 & 30 \\
\hline \hline Sim 1 & $\mathbf{3 . 4 3 6}$ & 4.522 & 3.620 & 5.400 & 9.501 & 10.019 \\
\hline Sim 2 & $\mathbf{1 . 0 7 5}$ & 5.884 & 3.975 & 10.185 & 7.102 & 3.331 \\
\hline Sim 3 & 2.470 & $\mathbf{0 . 0 5 1}$ & 0.168 & 0.287 & 0.324 & 0.509 \\
\hline Sim 4 & $\mathbf{0 . 2 0 8}$ & 0.836 & 0.804 & 3.004 & 3.444 & 3.067 \\
\hline Sim 5 & $\mathbf{0 . 2 7 8}$ & 0.935 & 0.837 & 1.777 & 1.790 & 7.534 \\
\hline \hline Mean & $\mathbf{1 . 4 9 3 5}$ & 2.445 & 1.880 & 4.130 & 4.432 & 4.890 \\
\hline Std & $\mathbf{1 . 4 1 7}$ & 2.585 & 1.774 & 3.868 & 3.796 & 3.813 \\
\hline
\end{tabular}

Table 2: Observed mean and standard deviation error (degrees) over all windows for a fixed window size $k$ (human dataset)

\begin{tabular}{|l|c|c|c|c|c|c|}
\hline$k$ & 5 & 10 & 15 & 20 & 25 & 30 \\
\hline \hline Subj 1 & $\mathbf{0 . 1 2 9}$ & 0.143 & 0.465 & 0.380 & 0.688 & 1.00 \\
\hline Subj 2 & $\mathbf{0 . 1 1 8}$ & 0.145 & 0.257 & 0.267 & 0.316 & 0.395 \\
\hline Subj 3 & $\mathbf{0 . 2 7 4}$ & 0.470 & 1.040 & 0.917 & 1.390 & 1.280 \\
\hline Subj 4 & 0.097 & 0.041 & $\mathbf{0 . 0 2 7}$ & 0.028 & 0.038 & - \\
\hline Subj 5 & 0.221 & $\mathbf{0 . 2 1 8}$ & 0.241 & 0.380 & 0.511 & 0.592 \\
\hline \hline Mean & $\mathbf{0 . 1 6 8}$ & 0.203 & 0.407 & 0.394 & 0.589 & 0.653 \\
\hline Std & $\mathbf{0 . 0 7 6}$ & 0.162 & 0.388 & 0.326 & 0.509 & 0.501 \\
\hline
\end{tabular}

We compare our method to a variety of state-of-the-art techniques [19, 4, 1, 26, 2], though all of these require more restrictive setups than our approach and are not able to operate in loosely constrained scenarios. However, they still provide a good reference for the proposed approach. Given the dearth of less constrained methods, we compared the reported mean calibration errors of these approaches with our own results.

In general, a window size of 5 frames gave the best performance for our method, with a mean accuracy of $1.493 \pm 1.417$ for the simulated dataset and $0.168 \pm 0.076$ for the human subject dataset. As shown in Figure 10, accuracy tends to decrease as window size increases, possibly due to change in the eye-camera model parameters over longer windows of observation. Our system achieved a performance of 4-5 fps with an unoptimized matlab implementation $^{1}$.

For comparison, we note that Pfeuffer et al. [19] achieve gaze estimation errors as low as 0.55 degrees by estimating the visual axis with 4 glints and having the user initially participate in active calibration procedure. Guestrin et al. [4] achieve an average gaze error of 0.9 degrees with one calibration point. Recent works such as Alnajar et al. [1] have

\footnotetext{
${ }^{1}$ Code is available at http://cs.unc.edu/ jmf/ publications/gaze_release.7z.
} 

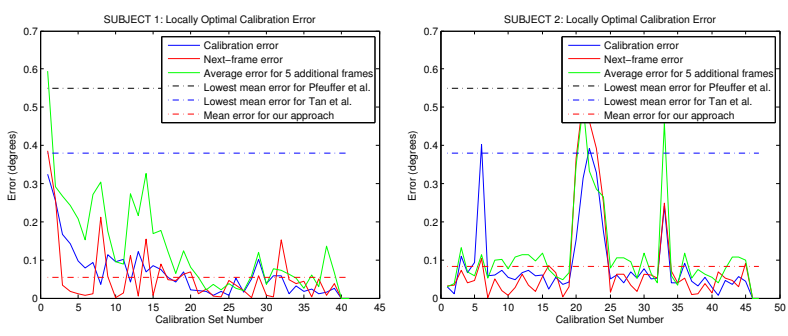

Figure 8: Different Calibration Errors for Subject 1 and Subject 2 using a window size of 5 .

produced calibration-free gaze estimation systems, but their errors are on the order of 1.3 to 7.8 degrees. Other calibration techniques such as Tan et al. [26] manage to report gaze estimation errors as low as 0.23 degrees but require over 250 calibration points; Chen et al. [2] reported quite impressive errors in order of 1.77 to 2.4 degrees without any active-calibration. Our system produces gaze estimates with significantly lower error than similar methods. In addition, similar to Chen et al. [2], our technique does not require any calibration before use. As such, our technique is very useful in real-world scenarios.

Figures 8 and 9 plot the gaze estimation errors w.r.t the observation window in time for real and simulated datasets. The solid lines show the calibration error for each window, for the next frame based on the previous window parameters, and for the five next frames. These measures of error give an idea of how well the proposed local gaze estimation technique works. As is evident in the figures, our approach was able to achieve quite reasonable accuracies. These results indicate that our continuous calibration scheme significantly outperforms a global calibration scheme for the same setup. Intuitively, these results imply that continuous recalculation of the eye-device calibration allows for a tighter fit to changes in the eye-device model over time. Generally, the performance of our method was consistent across multiple users who had different eye shapes and iris colors. The error for the human datasets was mainly due to large pupil occlusions and IR lighting inconsistencies, which would cause comparable errors in other gaze estimation systems. Note that a different user-facing camera orientation could reduce occlusion and improve performance further.

The results of our approach for Subjects 1 and 2 are plotted in Figure 8 along with the mean performances of stateof-the-art techniques. Table 1 shows how our system maintains high calibration accuracy for different window sizes used for calibration despite the head-worn device starting in an unknown pose (hence higher error in the start). As shown in Figure 10, the error estimation for the gaze detection system increases as the local window size is increased and the system converges to a global calibration performance.
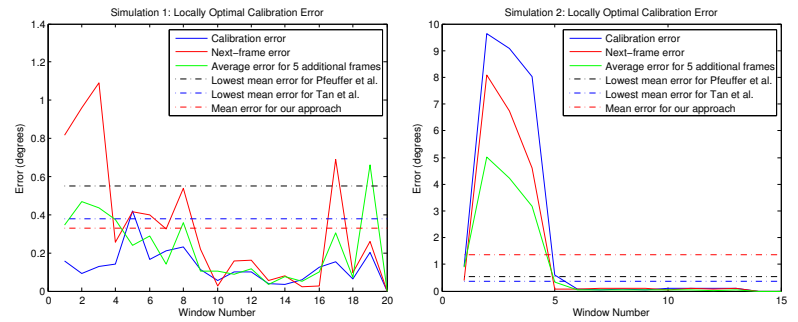

Figure 9: Different calibration errors for Simulation 1 and Simulation 2 using a window size of 10 .
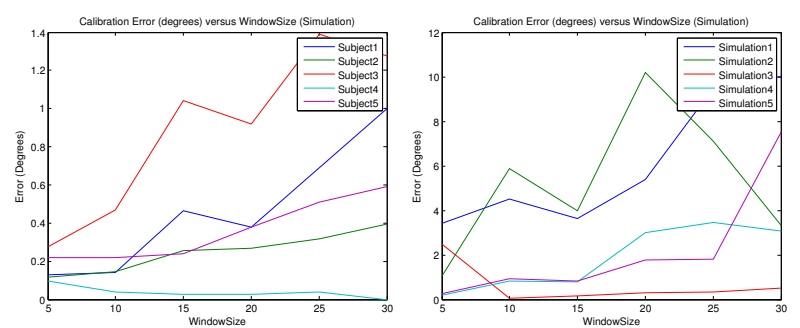

Figure 10: Plot of mean calibration error (degree) vs Window Size.

This is an effect of the continuously changing eye-device configuration due to the loose fit of the device, which cannot be explained by the global transformation frameworks. However, our continuous calibration approach handles this transformation by performing adaptive calibration over local windows and consistently outperforms the more restrictive setups of existing state of the art methods.

\section{Conclusion}

Gaze estimation techniques provide a wide array of new possibilities for head-worn devices and are in high-demand for providing additional user inputs to consumer-grade electronic systems. We believe that head-worn devices will be the next battleground for improving user interaction. In this paper, we proposed a novel, continuous, automatic calibration scheme which operates upon locally optimal models of the eye-device relationship at any given time. Calibration can be performed in a large number of unconstrained environments since our system relies upon natural calibration interest areas instead of traditional calibration markers. Our experiments demonstrate that our system produces highly accurate estimates of the user's gaze with respect to the user's scene, allowing for a head-worn device to update its calibration if the device-eye pose changes over time. Our results show that our continuous calibration technique is robust and can outperform state-of-the-art global calibration schemes by automatically adapting to variations in eyecamera pose. 


\section{Acknowledgment}

This material is based upon work supported by the National Science Foundation under Grant No CNS-1405847, NSF IIS 1423059, and US Army Research, Development and Engineering Command Grant No W911NF-14-1-0438. We would also like to thank True Price and Enrique Dunn for their insights and discussions.

\section{References}

[1] F. Alnajar, T. Gevers, R. Valenti, and S. Ghebreab. Calibration-free gaze estimation using human gaze patterns. In 15th IEEE International Conference on Computer Vision, 2013. 2, 3, 7

[2] J. Chen and Q. Ji. Probabilistic gaze estimation without active personal calibration. In Computer Vision and Pattern Recognition (CVPR), 2011 IEEE Conference on, pages 609616, June 2011. 2, 3, 7, 8

[3] F. Corno, L. Farinetti, and I. Signorile. A cost-effective solution for eye-gaze assistive technology. Multimedia and Expo, 2002. ICME '02. Proceedings. 2002 IEEE International Conference on, 2:433-436 vol.2, 2002. 3

[4] E. Guestrin and E. Eizenman. General theory of remote gaze estimation using the pupil center and corneal reflections. Biomedical Engineering, IEEE Transactions on, 53(6):1124-1133, June 2006. 4, 7

[5] D. Hansen and Q. Ji. In the eye of the beholder: A survey of models for eyes and gaze. Pattern Analysis and Machine Intelligence, IEEE Transactions on, 32(3):478-500, March 2010. 2, 3

[6] J. Harel, C. Koch, and P. Perona. Graph-based visual saliency. In ADVANCES IN NEURAL INFORMATION PROCESSING SYSTEMS 19, pages 545-552. MIT Press, 2007. 3, 5

[7] X. Hou, J. Harel, and C. Koch. Image signature: Highlighting sparse salient regions. IEEE Transactions on Pattern Analysis and Machine Intelligence, 34(1):194-201, 2012. 3, 5,7

[8] E. Jain, Y. Sheikh, and J. Hodgins. Inferring artistic intention in comic art through viewer gaze. In ACM Symposium on Applied Perception (SAP). 1

[9] R. Kumar, A. Ilie, J.-M. Frahm, and M. Pollefeys. Simple calibration of non-overlapping cameras with a mirror. In Computer Vision and Pattern Recognition, 2008. CVPR 2008. IEEE Conference on, pages 1-7, June 2008. 2, 6

[10] U. Lahiri, Z. Warren, and N. Sarkar. Design of a gazesensitive virtual social interactive system for children with autism. Neural Systems and Rehabilitation Engineering, IEEE Transactions on, 19(4):443-452, Aug 2011. 3

[11] U. Lahiri, Z. Warren, and N. Sarkar. Dynamic gaze measurement with adaptive response technology in virtual reality based social communication for autism. Virtual Rehabilitation (ICVR), 2011 International Conference on, pages 1-8, June 2011. 3

[12] V. Leborn Alvarez, A. Garca-Daz, X. Fdez-Vidal, and $\mathrm{X}$. Pardo. Dynamic saliency from adaptative whitening. In
Natural and Artificial Computation in Engineering and Medical Applications, volume 7931 of Lecture Notes in Computer Science, pages 345-354. Springer Berlin Heidelberg, 2013. 3, 5, 7

[13] F. Martinez, A. Carbone, and E. Pissaloux. Combining firstperson and third-person gaze for attention recognition. In Automatic Face and Gesture Recognition (FG), 2013 10th IEEE International Conference and Workshops on, pages 16, April 2013. 2, 3

[14] A. Meyer, M. Böhme, T. Martinetz, and E. Barth. A singlecamera remote eye tracker. In Proceedings of the 2006 International Tutorial and Research Conference on Perception and Interactive Technologies, PIT'06, pages 208-211, Berlin, Heidelberg, 2006. Springer-Verlag. 2

[15] C. Morimoto, A. Amir, and M. Flickner. Detecting eye position and gaze from a single camera and 2 light sources. In Pattern Recognition, 2002. Proceedings. 16th International Conference on, volume 4, pages 314-317 vol.4, 2002. 2, 3, 4

[16] A. Nakazawa and C. Nitschke. Point of gaze estimation through corneal surface reflection in an active illumination environment. In A. Fitzgibbon, S. Lazebnik, P. Perona, Y. Sato, and C. Schmid, editors, Computer Vision ECCV 2012, Lecture Notes in Computer Science, pages 159-172. Springer Berlin Heidelberg, 2012. 3

[17] H.-M. Park, S.-H. Lee, and J.-S. Choi. Wearable augmented reality system using gaze interaction. In Mixed and Augmented Reality, 2008. ISMAR 2008. 7th IEEE/ACM International Symposium on, pages 175-176, Sept 2008. 3

[18] H. S. Park, E. Jain, and Y. Sheikh. 3d social saliency from head-mounted cameras. In NIPS, pages 431-439, 2012. 1

[19] K. Pfeuffer, M. Vidal, J. Turner, A. Bulling, and H. Gellersen. Pursuit calibration: Making gaze calibration less tedious and more flexible. In Proceedings of the 26th Annual ACM Symposium on User Interface Software and Technology, UIST '13, pages 261-270, New York, NY, USA, 2013. ACM. 7

[20] B. Pires, M. Devyver, A. Tsukada, and T. Kanade. Unwrapping the eye for visible-spectrum gaze tracking on wearable devices. In Applications of Computer Vision (WACV), 2013 IEEE Workshop on, pages 369-376, Jan 2013. 2

[21] F. Pirri, M. Pizzoli, D. Rigato, and R. Shabani. 3d saliency maps. In Computer Vision and Pattern Recognition Workshops (CVPRW), 2011 IEEE Computer Society Conference on, pages 9-14, June 2011. 3

[22] F. Pirri, M. Pizzoli, and A. Rudi. A general method for the point of regard estimation in $3 \mathrm{~d}$ space. In Computer Vision and Pattern Recognition (CVPR), 2011 IEEE Conference on, pages 921-928, June 2011. 3

[23] K. Santner, G. Fritz, L. Paletta, and H. Mayer. Visual recovery of saliency maps from human attention in $3 \mathrm{~d}$ environments. In Robotics and Automation (ICRA), 2013 IEEE International Conference on, pages 4297-4303, May 2013. 3

[24] T. Shi, M. Liang, and X. Hu. A reverse hierarchy model for predicting eye fixations. CoRR, abs/1404.2999, 2014. 2, 3, 5,7 
[25] Y. Sugano, Y. Matsushita, and Y. Sato. Appearance-based gaze estimation using visual saliency. IEEE Transactions on Pattern Analysis and Machine Intelligence, 35(2):329-341, 2013. 3, 6

[26] K.-H. Tan, D. Kriegman, and N. Ahuja. Appearance-based eye gaze estimation. In Applications of Computer Vision, 2002. (WACV 2002). Proceedings. Sixth IEEE Workshop on, pages 191-195, 2002. 2, 7, 8

[27] T. Toyama, A. Dengel, W. Suzuki, and K. Kise. Wearable reading assist system: Augmented reality document combining document retrieval and eye tracking. In Document Analysis and Recognition (ICDAR), 2013 12th International Conference on, pages 30-34, Aug 2013. 3

[28] A. Tsukada, M. Shino, M. Devyver, and T. Kanade. Illumination-free gaze estimation method for first-person vision wearable device. In Computer Vision Workshops (ICCV Workshops), 2011 IEEE International Conference on, pages 2084-2091, Nov 2011. 3

[29] L. Twardon, H. Koesling, A. Finke, and H. Ritter. Gazecontingent audio-visual substitution for the blind and visually impaired. In Pervasive Computing Technologies for Healthcare (PervasiveHealth), 2013 7th International Conference on, pages 129-136, May 2013. 3

[30] A. Villanueva and R. Cabeza. A novel gaze estimation system with one calibration point. Systems, Man, and Cybernetics, Part B: Cybernetics, IEEE Transactions on, 38(4):11231138, Aug 2008. 3

[31] J. Wang, E. Sung, and R. Venkateswarlu. Eye gaze estimation from a single image of one eye. In Computer Vision, 2003. Proceedings. Ninth IEEE International Conference on, pages 136-143 vol.1, Oct 2003. 2 